Research Paper

\title{
Shockwave Targeting on Subchondral Bone Is More Suitable than Articular Cartilage for Knee Osteoarthritis
}

\author{
Wen-Yi Chou ${ }^{1,2}$, Jai-Hong Cheng ${ }^{2,3}{ }^{\bowtie}$, Ching-Jen Wang ${ }^{1,2}{ }^{\circledR}$, Shan-Ling Hsu ${ }^{1,2}$, Jen-Hung Chen ${ }^{1}$, Chien-Yiu \\ Huang 1,2 \\ 1. Section of Sports Medicine, Department of Orthopedic Surgery, Kaohsiung Chang Gung Memorial Hospital and Chang Gung University College of \\ Medicine, Kaohsiung, Taiwan. \\ 2. Center for Shockwave Medicine and Tissue Engineering, Kaohsiung Chang Gung Memorial Hospital and Chang Gung University College of Medicine, \\ Kaohsiung, Taiwan. \\ 3. Medical Research, Kaohsiung Chang Gung Memorial Hospital and Chang Gung University College of Medicine, Kaohsiung, Taiwan.
}

$\square$ Corresponding authors: Ching-Jen Wang, M. D. Department of Orthopedic Surgery, Kaohsiung Chang Gung Memorial Hospital, 123 Tai-Pei Road, Niao Sung District, Kaohsiung, Taiwan 833. Tel.: 886-7-733-5279; Fax: 886-7-733-5515; Email: w281211@adm.cgmh.org.tw or Jai-Hong Cheng, Ph. D. Center for Shockwave Medicine and Tissue Engineering, Department of Medical Research, Kaohsiung Chang Gung Memorial Hospital, 123 Tai-Pei Road, Niao Sung District, Kaohsiung, Taiwan 833. Fax: +886-7-7317123 ext 8150. Tel: +886-7-731-7123 ext 6422. E-mail address: jh1106520@gmail.com

(c) Ivyspring International Publisher. This is an open access article distributed under the terms of the Creative Commons Attribution (CC BY-NC) license (https://creativecommons.org/licenses/by-nc/4.0/). See http://ivyspring.com/terms for full terms and conditions.

Received: 2018.04.13; Accepted: 2018.11.29; Published: 2019.01.01

\begin{abstract}
Our study compared the effects of extracorporeal shockwave therapy (ESWT) on the subchondral bone and the articular cartilage in the treatment of early osteoarthritis (OA) of rat knee. The rats were divided into 5 groups which included Sham group, Meniscus group (ESWT applied on medial meniscus), OA group (arthrotomy and medial menisectomy (MMx) and anterior cruciate ligament transection (ACLT), $\mathrm{T}(\mathrm{M})$ group (arthrotomy and MMx and ACLT followed by ESWT on medial tibial subchondral bone) and Articular cartilage group (arthrotomy and MMx and ACLT followed by ESWT on medial articular cartilage). Evaluations included the pathological changes of the synovium, articular cartilage and subchondral bone, and compared with ESWT on the meniscus, medial tibial subchondral bone and articular cartilage. The ESWT $\left(0.25 \mathrm{~mJ} / \mathrm{mm}^{2}\right.$ and 800 impulses $)$ did not cause any damages on the cartilage of the meniscus and the tissue of the joint when compared with Sham group. Among the treatment of osteoarthritic groups (OA, T(M) and Articular cartilage groups), $\mathrm{T}(\mathrm{M})$ group showed significant in pathological examination, micro-CT analysis, cartilage grading score and grading of synovium changes by compared with OA and Articular cartilage groups $(P<0.05)$ in the treatment of early OA knee. In immunohistochemical analysis, $T(M)$ group significantly increased the expression of TGF- $\beta 1$ but reduced DMP-1, MMP-13 and ADAMTS-5 in the cartilage by compared with OA group and Articular cartilage group $(P<0.05)$. Our results showed that subchondral bone was an excellent target than articular cartilage for ESWT on early knee osteoarthritis.
\end{abstract}

Key words: extracorporeal shock wave therapy, osteoarthritis, articular cartilage, knee, tissue regeneration

\section{Introduction}

Osteoarthritis (OA) knee is caused by damage of cartilage and underlying bone. OA knee is the most common cause of chondral lesions after age forty. Degenerative lesions are of different depths and shapes. This damage can accumulate over time and lead to pain and stiffness in the joints. Stiffening of subchondral bone results in less shock absorption and cartilage matrix breakdown [1]. The pathological feature of OA knee has been considered to cause from a significant inflammation of articular and peri-articular structures and destruction in the cartilage organization [2-5]. The weight bearing also enlarges the lesion and abrades the subchondral bone over time [1]. Loss of biomechanical function due to meniscal tears and loss of knee stability due to ligamentous damage (particularly the anterior cruciate ligament) result in increased cartilage injury [6]. Therefore, it is believed that the progression of OA knee involves with many factors, including articular cartilage destruction, fraction and sclerosis in the 
subchondral bone, bone cyst, and osteophyte formation, deterioration of muscular function and inflammation in the synovium and tendon of the knee $[7,8]$. The treatment of OA knee depends on several specific factors, by the selection criteria of patients, daily life with activities, age of patients, the cause of the disease, and the grading of lesions $[9,10]$. Although total knee replacement poses satisfactory rate from 81 to $90.3 \%$, either nonsurgical treatment or joint preserving procedure are still favored before arthroplasty [11-13]. For the early OA, a nonsurgical treatment includes weight reduction, strengthening, low-impact aerobic exercise, neuromuscular education and anti-inflammation medicine, platelet-rich plasma (PRP) injection [9]. Joint preserving procedures are usually preserved when it failed to respond to the nonsurgical treatment, and it includes arthroscopic lavage and debridement, abrasion arthroplasty, subchondral drilling, microfracture, osteochondral autografting, osteochondral allografting, autologous chondrocyte implantation (ACI), matrix-induced ACI (MACI), artificial chondroplasty and bioscaffolds, gene therapy [14-16]. On account of non-invasive nature the emerging application of extracorporeal shockwave therapy (ESWT) on OA knee is gradually being noticed [17].

ESWT has shown success in the treatment of many musculoskeletal disorders, including plantar fasciitis of the heel, calcific tendonitis of the shoulder, lateral epicondylitis of the elbow and non-union of long bone fracture [17-19]. ESWT was shown to induce biological responses by stimulating the ingrowth of neovascularization associated with up-regulations of angiogenesis and osteogenesis growth factors that lead to tissue regeneration and repair at the achilles tendon-bone junction in animal experiments [19]. Previous reports also showed that the mechanotransduction is the major pathway by ESWT to induce the biological responses of angiogenetic and tissue regeneration mechanism at cellular and molecular levels that produces therapeutic effects in clinical application [20,21]. For the treatment of OA knee, documents indicate that subchondral bone of medial tibia is the key target for ESWT and has good effect as compared with the different locations of knee [22,23]. However, most regeneration treatments in $\mathrm{OA}$ knee aimed on the articular cartilage. Yet, the exact effectiveness of ESWT on the articular cartilage of OA knee is unclear. Therefore, we conducted a comparative animal study to clarify the effectiveness and safety of ESWT on articular cartilage of medial compartment and subchondral bone of medial tibial of OA knees.

\section{Materials and Methods}

\section{The experimental design}

Fifty, 8-week-old female Sprague-Dawley (SD) rats were purchased from BioLASCO (Taipei, Taiwan) and properly maintained for the experiment. The IACUC protocol of the animal study was approved by the Animal Care Committee of Kaohsiung Chang Gung Memorial Hospital. All rats were maintained at the Laboratory Animal Center (Kaohsiung Chang Gung Memorial Hospital, Kaohsiung, Taiwan) for 1 week before the experiments. They were housed at 23 $\pm 1{ }^{\circ} \mathrm{C}$ with a 12-hour light and dark cycle as well as given food and water. The SD rats were randomly divided into five groups, which were composed of 10 rats in each group (Fig. 1A). The group I was designated as Sham. They received sham arthrotomy of left knee without an anterior cruciate ligament transacted (ACLT) and medial meniscectomy (MMx). Group II was designated as Meniscus. They received sham arthrotomy of left knee without ACLT and MMx of the left knee, and then the shockwave applied to the medial edge of the meniscus. Group III was designated as OA. The animals received ACLT and MMx of left knee. Group IV was designated as T(M). Rats received ACLT and MMx of left knee and the shockwave applied to the proximal medial tibia plateaus. Group V was designated as Articular cartilage. The animals received ACLT and MMx of left knee and the shockwave applied to the articular cartilage surface of the proximal medial tibia plateaus. At 12-weeks post-surgery, the animals were scarified and the knees were collected from experiments.

\section{Surgical procedure}

The left knee was prepared in a surgically sterile fashion. Through mini-arthrotomy, the ACL was transected with a scalpel, and MM was performed by excising the entire medial meniscus. The knee joint was irrigated, and the incision was closed. Prophylactic antibiotics with ampicillin, $50 \mathrm{mg} / \mathrm{kg}$ body weight were given for 5 days after surgery. Postoperatively, the animals were cared by a veterinarian and the surgical sites and the activities of animals were observed daily.

\section{Application of shockwave}

In ESWT groups, ESWT was performed in the first week after surgery when the knee operation wounds healed [24]. The animals were sedated with 1:1 volume mixture of Rompun $(5 \mathrm{mg} / \mathrm{kg})+$ Zoletil (20 mg/kg) while receiving ESWT. Ultrasound guidance (Toshiba Medical Systems Corporation, Tokyo, Japan) was performed before ESWT and allowed for precise tracking of the anatomical 
locations of ESWT application. The source of shockwave was from the DUOLITH® SD1 »ultra« (Storz Medical, Switzerland) and the foci of shockwave application were chosen on the target region using the ultrasound machine (Supplemental Figure 1). For Meniscus group, each animal received ESWT applied to the medial joint of left knee where the medial edge of the meniscus. For $\mathrm{T}(\mathrm{M})$ group, each animal received ESWT applied on the exact location at $0.5 \mathrm{~cm}$ from the joint lines and on tibia 0.5 $\mathrm{cm}$ from medial lateral skin horizontally [25]. For Articular cartilage group, each animal was placed in a supine position with the left knee flexed at maximum angle to expose the cartilage surface of tibia plateau. Application of 800 impulses of shockwave at 0.25 $\mathrm{mJ} / \mathrm{mm}^{2}$ energy flux density was performed on the medial femoral condyle. The dosage was modified based on our previous studies [22,23]. After ESWT, the animals were cared for by the veterinarian until scarification.

\section{Bone mineral density analysis}

The bone mineral density values within the region of interests (ROI) in the medial proximal tibia and distal femur condyles of the joint samples were measured by using dual-energy X-ray absorptiometry (DEXA) (Hologic QDR $4500 \mathrm{~W}$, Hologic, USA) at a pixel area resolution at $640 \mu \mathrm{m}^{2}$.

\section{The measurements of OA lesion score and tibia lesion area}

All knee joints were separated into the femur and tibia portions and were examined under a magnification scope (Carl Zeiss, Germany). The gross pathologic lesions with arthritic changes on femoral condoyle and tibial plateau were identified and quantified separately by the semi-quantitative scale [26]. The severity of joint-surface damage was categorized and scored as follows: (a) Intact surface or normal in appearance $=0$ point, $(b)$ surface rough with minimal fibrillation or a slight yellowish discoloration $=1$ point, $(\mathrm{c})$ cartilage erosion extending into the superficial or middle layers $=2$ points, $(\mathrm{d})$ cartilage erosion extending into the deep layers $=3$ points, (e) complete cartilage erosion with subchondral bone exposed $=4$ points. The average OA lesion score was obtained by summing up the average scores of the femur and tibia from each animal and divided by the number of animals per group.

For the percent lesion area measurements, the total surfaces of osteophye and lesion on medial tibia plateaus were manually traced by using ImageJ software program (NIH, USA) and areas were determined by using the ImagePro Plus analysis program (Media Cybernetics Inc. USA) [27]. Percentages of osteophyte and lesion areas were calculated as osteophyte and lesion areas divided by medial tibia plateau areas $\times 100 \%$.

\section{Micro-CT analysis}

The proximal part of the tibia and the distal part of the femur were scanned by micro-CT scanner (Skyscan 1076; Skyscan, Luxembourg, Belgium) with isotropic voxel size of $36 \times 36 \times 36 \mu \mathrm{m}^{3}$, as previously described [28]. The X-ray voltage was set at $100 \mathrm{kV}$, and the current, at $100 \mu \mathrm{A}$. X-ray projections were obtained at 0.75 -degrees angular step with a scanning angular range of 180 degrees. Reconstructions of the image slices were performed with NRecon software (Skyscan), and the process generated a series of planar transverse gray value images. Volumes of interest (VOI) of bone graphics were reconstructed with a semi-automatic contouring method by Skyscan CT-Analyser Software (Skyscan) and parameters of bone volume $(\%)$, porosity $(\%)$, trabecular thickness $(\mu \mathrm{m})$, and the traceulcar number per $\mathrm{mm}$ were calculations of the femurs and tibias. The subchondral plate thicknesses of femur and tibia were measured by DataViewer software (Skyscan). The subchondral plate thickness of each section was defined as the average value of three manual thickness measurements on lateral, medial and peripheral area. Four digital images of the middle sagittal plane sections from femur and tibia were used for thickness histomorphometry analysis.

\section{Modified Mankin score and cartilage thickness measurements}

The degenerative changes of the cartilage were graded histologically by using the modified Mankin Score to assess the severity of OA via Safranin O stain. The scoring system included the cartilage surface damage, loss of celluarlity, loss of matrix staining, loss of tidemark integrity and proportions of the lesion site. The modified Mankin scores were obtained on a 0 to 33 scale in addition to the analytical factors [29]. The cartilage thicknesses were measured by eight non-consecutive sections, which obtained at $100 \mu \mathrm{m}$ intervals were measured per knee joint. Safranin-O stain which provided the layer discrimination between un-calcified cartilage (UCC) and calcified cartilages (CC). Cartilage areas were automatically calculated by ImageJ software and average thicknesses were then determined as areas divided by lengths. The UCC and CC thicknesses were reconfirmed by measuring individual cartilage point-to-point distance by averaging at least six measurements per sample. 


\section{Synovitis scoring and IL- $1 \beta$ layer scoring}

Haematoxylin and eosin stainings were performed in evaluating synovitis by scoring the thickening of the synovial lining, cellular hyperplasia and infiltration into joint cavity and synovium. A scheme for the histopathological assessment of the three features of chronic synovitis was described in detail and score ranks were defined as: $0-1=$ no synovitis; $2-4=$ low grade synovitis; $5-9=$ high grade synovitis [30]. For IL-1 $\beta$ layer scoring, the semiquantitative scale of IL-1 $\beta$ expression by IHC on synovial cell layers was stated as: $0=$ no staining; $1=$ $1-10 \%$ positively staining cells; $2=11-25 \%$ positively staining cells; $3=26-50 \%$ positively staining cells; $4=$ $51-75 \%$ positively staining cells; $5=76-100 \%$ [31].

\section{Immunohistochemical analysis}

The harvested knee specimens were fixed in $4 \%$ formaldehyde solution in PBS for 48 hours and decalcified with $10 \%$ EDTA in 0.1M PBS. Decalcified tissues were embedded in paraffin wax. The specimens were cut longitudinally into $5 \mu \mathrm{m}$ thick sections and transferred to polylysine-coated slides (Thermo Fisher Scientific, USA). The immunohistochemical stains were performed by following the protocol from the operation illustration of immunostaining kit (Abcam, USA). The tissue sections were deparaffinized with xylene, hydrated with a graded ethanol solution, and treated with a peroxide block and protein-blocking reagents. Sections of the specimens were stained with a specific antibody for IL-1 $\beta$ at 1:100 (Santa Cruz Biotechnology, USA), DMP-1 (LifeSpan BioSciences Inc., USA) at 1:200 dilution, TGF- $\beta 1$ at 1:200 (SpringBio, USA), MMP-13 at 1:200 (EnoGene, USA) and ADAMTS-5 at 1:100 (Abcam) for overnight to identify the chondrogenesis and chondrodegradation biomarkers. The immunoreactivity in specimens was demonstrated by using a goat anti-rabbit horseradish peroxidase (HRP)-conjugated and 3', 3'-diaminobenzendine (DAB), which were provided in the kit. The immunoactivities were quantified from five random areas in three sections of the same specimen by using a Zeiss Axioskop 2 plus microscope (Carl Zeiss, Germany). All images of each specimen were captured by using a cool CCD camera (Media Cybernetics, USA). The images were analyzed by using ImageJ analysis software (NIH, USA) for obtaining percent of positively staining cells.

\section{Statistical analysis}

SPSS ver. 17.0 (SPSS Inc., USA) was used in statistical analysis. Calculated data were expressed as mean \pm SD and One-way ANOVA with Tukey tests for post hoc (normal distribution) were used for group comparisons. Ranking data (non-normal distribution) was used Kruskal-Wallis test for comparisons of multiple groups.

\section{Results}

\section{ESWT applied on the surface of articular cartilage of OA knee}

There are many studies that has been assessed the effects of ESWT on different locations of OA knee $[22,23]$. However, the comparing effect of ESWT on the subchondral bone and surface of articular cartilage of OA knee are still unclear. In this study, ESWT was applied on the surface of articular cartilage of medial tibia (Articular cartilage group) to treatment of the OA rat knee by comparing with Sham, Meniscus, OA and $\mathrm{T}(\mathrm{M})$ groups (subchondral bone) (Figure 1A and Supplemental fig. 1). The pathological data showed that $\mathrm{T}(\mathrm{M})$ were significant changes than Articular cartilage group in OA lesion score $(1.60 \pm 0.21$ vs. $3.00 \pm 0.23 ; \mathrm{P}<0.05)$, maximum extension angle $(26.22 \pm 4.00$ vs. $57.36 \pm 8.67 ; \mathrm{P}<0.05)$, bone marrow density $(0.34 \pm 0.03$ vs. $0.29 \pm 0.02 ; \mathrm{P}<0.05)$, and medial tibia lesion $(55.43 \pm 8.32$ vs. $87.04 \pm 6.82 ; \mathrm{P}<0.05)$ by compared with OA group $(3.40 \pm 0.20,59.36 \pm 9.04$, $0.27 \pm 0.01$, and $80.93 \pm 10.46$ ) (Figure 1 and Table 1 ). In contrast to the surface of articular cartilage, ESWT on the subchondral bone improved the regeneration of tissue in the progression of OA changes.

In order to reveal the safety of ESWT dosage applied on the subchondral bone and the cartilaginous tissue of OA knee. ESWT $\left(0.25 \mathrm{~mJ} / \mathrm{mm}^{2}\right.$; 800 impulses) was applied to the medial meniscus of the rat knee (Figure 1A). We found that there were no significant differences between Sham and Meniscus groups in $\mathrm{OA}$ lesion score $(0.00 \pm 0.00$ versus $0.05 \pm$ $0.05)$, maximum extension angle (19.12 \pm 1.65 versus $19.91 \pm 1.87)$, bone marrow density $(0.35 \pm 0.02$ versus $0.34 \pm 0.04)$, and medial tibia lesion $(0.00 \pm 0.00$ versus $0.00 \pm 0.00$ ), respectively (Table 1 ). This data showed that the dosage of ESWT that we used did not cause any damages to cartilage and subchondral bone (Figure 1B and Figure 2).

\section{Bone mineral density and micro-CT analysis}

The bone mineral density (BMD) values within the region of interests (ROI) in the medial proximal tibia and distal femur condyles of the joint were presented in Figure 2 and Table 1. These results demonstrated that the BMD of $\mathrm{T}(\mathrm{M})$ group significantly increased $(0.34 \pm 0.03)$ and the medial tibia lesion decreased $(55.43 \pm 8.32)$ in the tibia compared with OA $(0.27 \pm 0.01$ and $80.93 \pm 10.46)$ and Articular cartilage $(0.29 \pm 0.02$ and $87.04 \pm 6.82)$ groups (both $P<0.05$ ). 


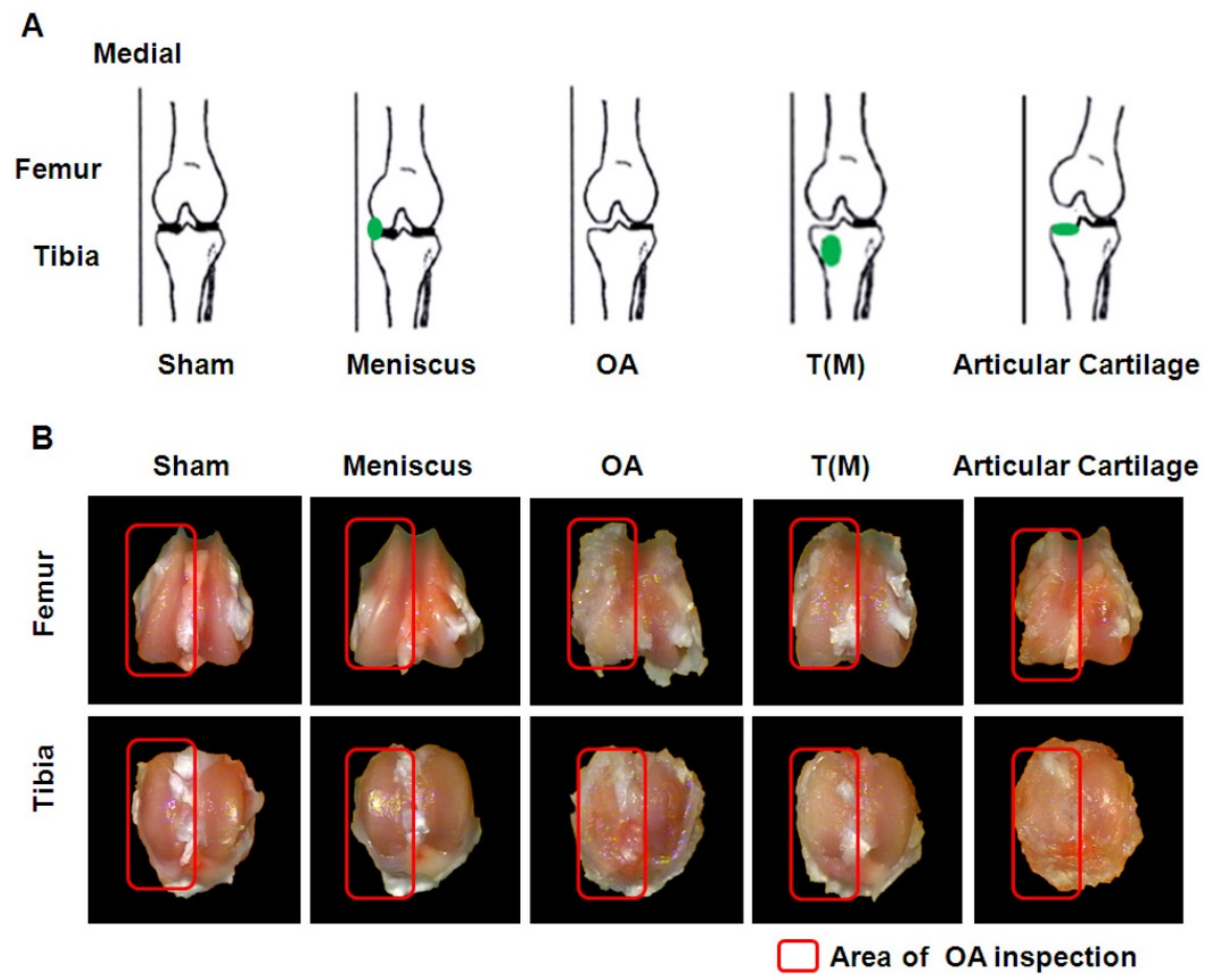

Figure 1. The experimental design and comparisons of gross appearance of femoral condlyles and tibial plateaus. (A) Sketches of left knee showed the specific locations of ESWT application (green dots) in an anteroposterior view. (B) Gross observation of osteoarthritic severity in transverse views of femur (upper panel) and tibia (lower panel) after 12 weeks post-treatment. Osteoarthritic wears on medial compartments of knee became increasingly more severe in OA and Articular cartilage groups than the T(M) group. The osteoarthritic wears were not observed in Sham and Meniscus groups. The magnification of the image was $\times 2$. All rats were $n=10$.

Table 1. Pathological and micro-CT analysis.

\begin{tabular}{|c|c|c|c|c|c|c|}
\hline Analysis & & Sham & Meniscus & $\mathrm{OA}$ & $\mathrm{T}(\mathrm{M})$ & Articular cartilage \\
\hline \multicolumn{7}{|l|}{ Pathological analysis } \\
\hline OA lesion score & & $0.00 \pm 0.00^{\mathrm{bc}}$ & $0.05 \pm 0.05^{\mathrm{bc}}$ & $3.40 \pm 0.20^{\mathrm{ac}}$ & $1.60 \pm 0.21^{\mathrm{ab}}$ & $3.00 \pm 0.23^{\mathrm{ac}}$ \\
\hline Maxima extension angle & & $19.12 \pm 1.65^{\mathrm{b}}$ & $19.91 \pm 1.87 \mathrm{~b}$ & $59.36 \pm 9.04^{\mathrm{ac}}$ & $26.22 \pm 4.00^{\mathrm{b}}$ & $57.36 \pm 8.67 \mathrm{ac}$ \\
\hline $\operatorname{BMD}\left(\mathrm{g} / \mathrm{cm}^{2}\right)$ & & $0.35 \pm 0.02^{\mathrm{b}}$ & $0.34 \pm 0.04^{\mathrm{b}}$ & $0.27 \pm 0.01^{\text {ac }}$ & $0.34 \pm 0.03^{b}$ & $0.29 \pm 0.02^{\mathrm{ac}}$ \\
\hline Medial Tibia lesion (\%) & & $0.00 \pm 0.00^{\mathrm{bc}}$ & $0.00 \pm 0.00^{\mathrm{bc}}$ & $80.93 \pm 10.46^{\mathrm{ac}}$ & $55.43 \pm 8.32^{\mathrm{ab}}$ & $87.04 \pm 6.82^{\mathrm{ac}}$ \\
\hline \multicolumn{7}{|l|}{ Micro-CT analysis } \\
\hline \multirow[t]{2}{*}{ Sb.Th $(\mu \mathrm{m})$} & Femur & $296.15 \pm 16.16$ & $294.43 \pm 8.50$ & $311.32 \pm 58.88$ & $284.51 \pm 20.93$ & $304.19 \pm 41.63$ \\
\hline & Tibia & $327.51 \pm 11.05^{\mathrm{b}}$ & $330.98 \pm 18.60$ & $260.71 \pm 46.30^{\mathrm{ac}}$ & $332.41 \pm 20.55^{b}$ & $301.66 \pm 50.00$ \\
\hline \multirow[t]{2}{*}{$\mathrm{BV} / \mathrm{TV} \%$} & Femur & $58.66 \pm 2.31^{b}$ & $58.52 \pm 1.42^{\mathrm{b}}$ & $48.79 \pm 5.30 \mathrm{ac}$ & $59.29 \pm 4.63^{b}$ & $50.27 \pm 4.24$ ac \\
\hline & Tibia & $58.85 \pm 2.21^{\mathrm{b}}$ & $58.88 \pm 2.01^{b}$ & $44.19 \pm 4.14^{\mathrm{ac}}$ & $62.59 \pm 2.44^{b}$ & $45.65 \pm 3.66^{\mathrm{ac}}$ \\
\hline \multirow[t]{2}{*}{ Tb.Th $(\mu \mathrm{m})$} & Femur & $149.75 \pm 4.74^{c}$ & $143.82 \pm 5.64^{c}$ & $157.48 \pm 21.20$ & $164.58 \pm 7.37 \mathrm{a}$ & $162.54 \pm 20.57$ \\
\hline & Tibia & $146.07 \pm 8.50^{\mathrm{bc}}$ & $150.85 \pm 7.81^{\mathrm{bc}}$ & $193.65 \pm 17.06^{\mathrm{a}}$ & $187.85 \pm 13.35^{\mathrm{a}}$ & $200.87 \pm 19.27 \mathrm{a}$ \\
\hline \multirow[t]{2}{*}{ Tb.N (1/mm) } & Femur & $4.03 \pm 0.22 \mathrm{bc}$ & $4.02 \pm 0.29 \mathrm{bc}$ & $2.97 \pm 0.53 \mathrm{a}$ & $3.35 \pm 0.26^{\mathrm{a}}$ & $2.60 \pm 0.41^{\mathrm{ac}}$ \\
\hline & Tibia & $3.94 \pm 0.31^{\mathrm{bc}}$ & $4.04 \pm 0.40^{\mathrm{bc}}$ & $2.07 \pm 0.21^{\mathrm{ac}}$ & $2.89 \pm 0.38^{\mathrm{ab}}$ & $2.07 \pm 0.28 \mathrm{ac}$ \\
\hline \multirow[t]{2}{*}{ Porosity (\%) } & Femur & $41.34 \pm 2.07 \mathrm{~b}$ & $41.48 \pm 1.27 \mathrm{~b}$ & $51.21 \pm 4.74^{\mathrm{ac}}$ & $40.71 \pm 4.14^{\mathrm{b}}$ & $49.73 \pm 3.79 \mathrm{ac}$ \\
\hline & Tibia & $41.15 \pm 2.21^{\mathrm{b}}$ & $41.12 \pm 2.01^{\mathrm{b}}$ & $55.81 \pm 4.14^{\mathrm{ac}}$ & $37.41 \pm 2.44^{\mathrm{b}}$ & $54.35 \pm 3.66^{\mathrm{ac}}$ \\
\hline
\end{tabular}

Mean \pm SD obtained for all experimental groups with statistical results by one-way ANOVA, except OA lesion scores were expressed as mean \pm SEM with statistical results by Kruskal-Wallis test. Statistical significance $\mathrm{p}<0.05$ : $\mathrm{a}=$ Sham vs. other groups; $\mathrm{b}=\mathrm{OA}$ vs. other groups; $=\mathrm{T}(\mathrm{M})$ vs. other groups. Sb.Th=Subchondral plate thickness, $\mathrm{BV}=$ Bone volume, $\mathrm{TV}=\mathrm{Tissue}$ volume, $\mathrm{Tb}$. Th=Trabecular bone thickness, $\mathrm{Tb} . \mathrm{N}=$ Trabecular bone number.

According to the micro-CT analysis, the sagittal and transverse plane section images of femur and tibia in knees showed differences in trabecular micro-architecture among the various treatment groups as represented in Figure 2. The subchondral bone cysts were clearly observed in the femur and tibia of OA (8 cysts were observed in 10 knees) and Articular cartilage groups (7 cysts were observed in 10 knees) (Figure 2A and 2B: red arrows) unless T(M) group. Analysis of the representative samples indicated that $\mathrm{OA}$ and Articular cartilage groups resulted in the deterioration of the trabecular bone micro-architecture, as demonstrated by the reduced Sb.Th, BV/TV, Tb.Th, and Tb.N, compared with the Sham group in subchondral bone of femur and tibia (obviously in the tibia, $P<0.05$ ) (Table 1 ). In contrast, porosity was significantly increased in response to $\mathrm{OA}$ and Articular cartilage groups as compared with the Sham group $(P<0.05)$. Obviously, ESWT on T(M) group significantly improved all the micro-architecture than $\mathrm{OA}$ and Articular cartilage groups $(P<0.05)$ (Table 1$)$. 
A
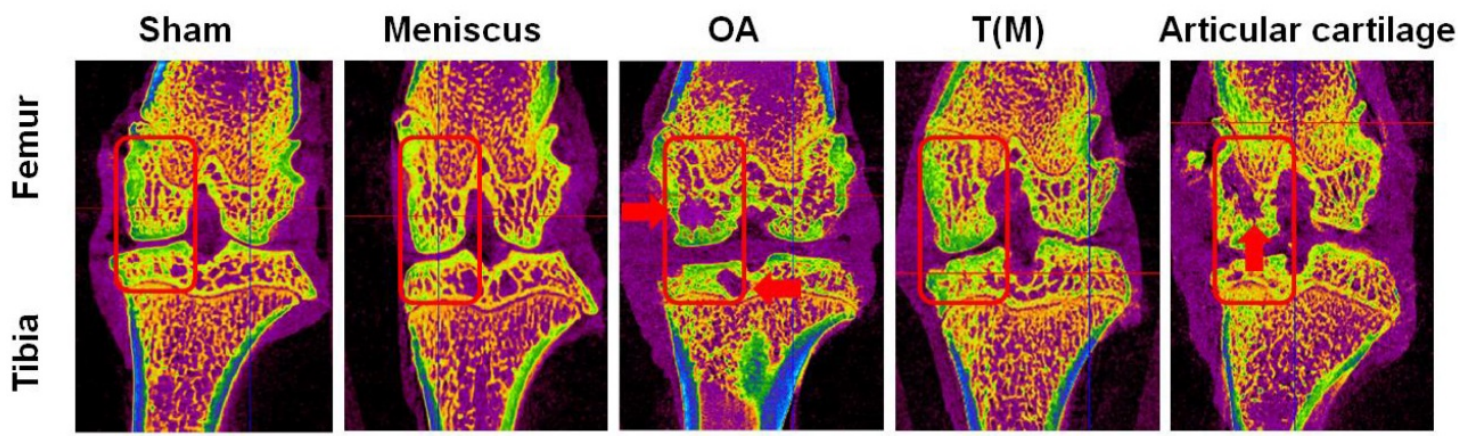

B

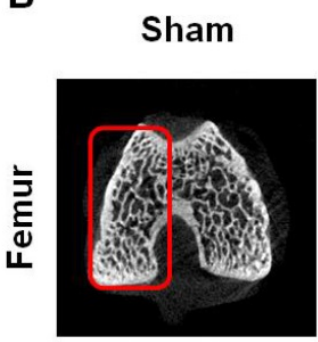

Meniscus

$O A$

$\mathrm{T}(\mathrm{M})$

\section{Articular cartilage}
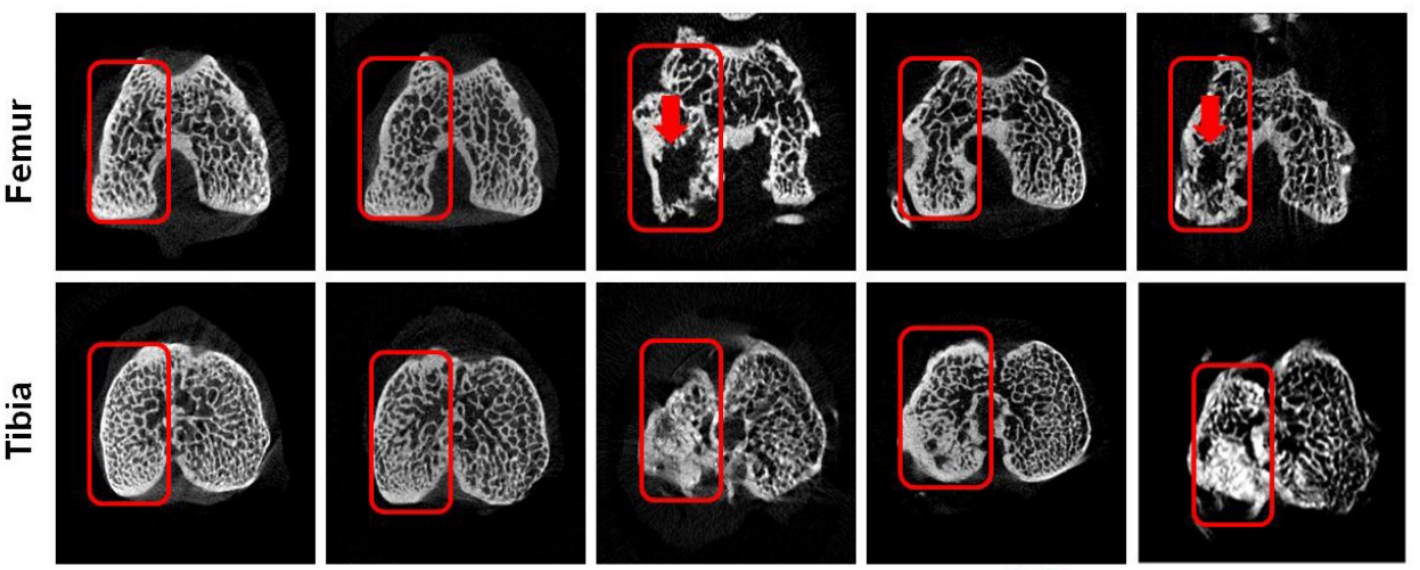

Area of $O A$ inspection

Figure 2. The micro-CT analysis of distal femur and proximal tibia in different groups. The sagittal (A) and transverse (B) views of micro-CT images showed the changes of osteoarthritic differences in subchondral bone structures of medial femoral and tibial compartments. The images with severe bone erosion, cyst and bone sclerosis were observed in OA and Articular cartilage groups, whereas $T(M)$ group showed mild to moderate sclerotic bone formation which was due to bone remodeling and regeneration. The region of interesting (ROI) was indicated in red rectangle. The red arrow was indicated bone cyst. All rats were $\mathrm{n}=10$.

\section{Analysis of articular cartilage integrity}

The normal functions of the articular cartilage depend on the integrity of the molecular composition in the cartilage extracellular matrix. However, OA causes the loss of integrity of articular cartilage of damage and degradation of cartilage extracellular matrix. As previous reports, ESWT on T(M) is reported to have the chondroprotective effect on articular cartilage [22,32]. Here, we applied ESWT on $\mathrm{T}(\mathrm{M})$ and compared with the surface of articular cartilage to elucidate the both treatment effects in the OA knee. The changes of cartilage compositions were measured by Safarine-O stain. The data showed that Sham, Meniscus and $\mathrm{T}(\mathrm{M})$ groups have the statistically significant difference in the cartilage damage, loss of cellularity, loss of matrix staining, loss of tidemark integrity and modified Mankin score compared with OA and Articular cartilage groups ( $P$ $<0.05$ ) (Figure 3 and Table 2).
The changes in the thickness of both uncalcified and calcified cartilage were measured and observed in the Sham, Meniscus, OA, T(M) and Articular cartilage groups. Sham, Meniscus and T(M) groups of sagittal sections of articular cartilage showed the intact superficial, mid, and deep zones (Figure 3: Cartilage integrity). The uncalcified and calcified thicknesses were obvious to define in these groups (Table 2). In contrast, $\mathrm{OA}$ and Articular cartilage groups showed the development of vertical fissures, loss of glycosaminoglycans, formation of chondrocyte clusters and matrix erosion (Figure 3: Cartilage integrity and Table 2). The structural components of the cartilage of $\mathrm{OA}$ and Articular cartilage groups were obviously indicative of abnormal repair processes. In addition, the calculation of uncalcified and calcified thicknesses became meaningless. The results demonstrated that ESWT on the subchondral bone was better than the surface of articular cartilage to improve the regeneration of cartilage and extracellular matrix in OA knee. 


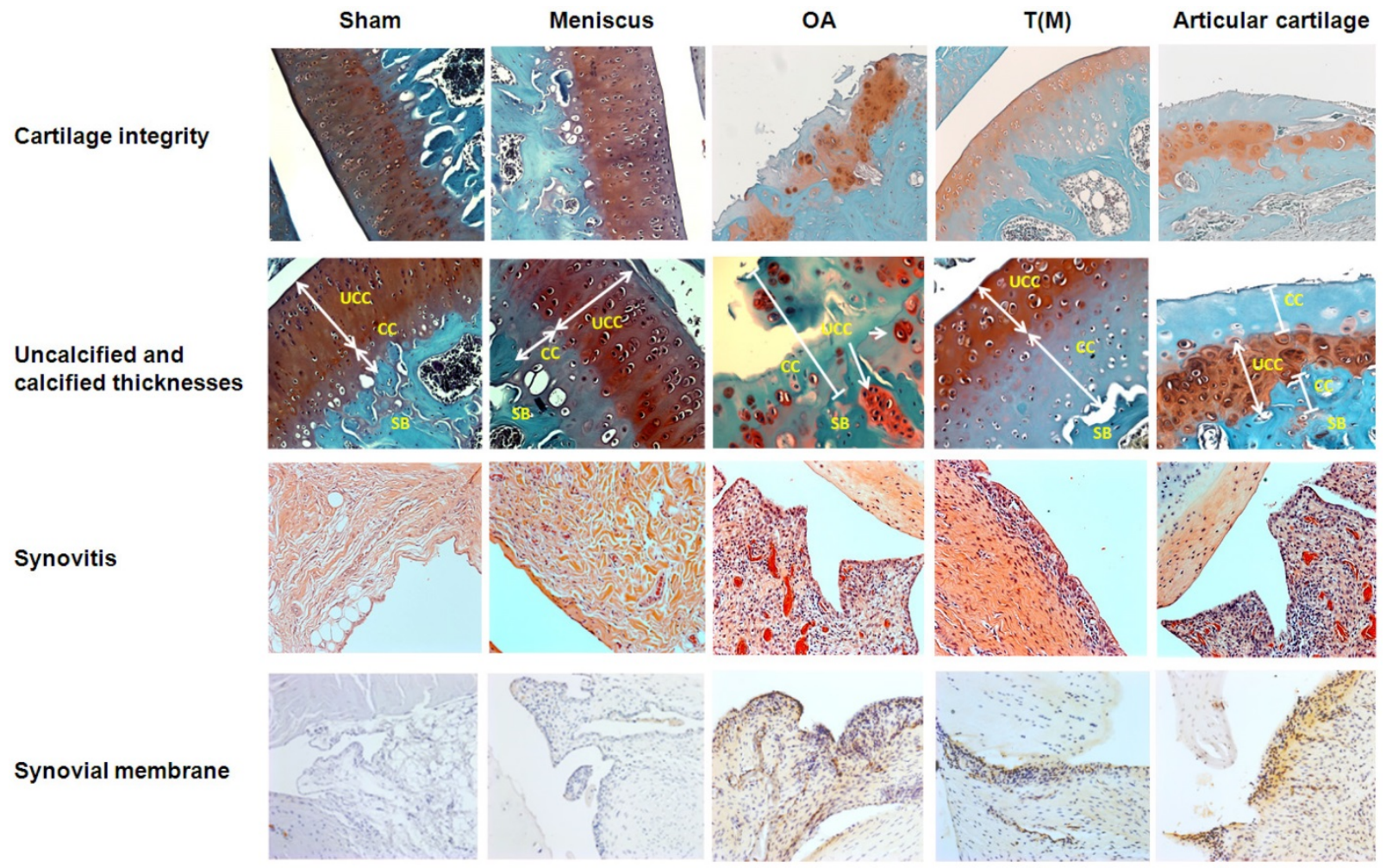

Figure 3. The analysis of cartilage and synovial membrane. For the cartilage integrity, $O A$ and Articular cartilage groups displayed more severe damage of cartilage as compared with other groups by Safranin-O staining. The magnification of the image was $\times 200$. In the measurements of UCC and CC thicknesses, OA and Articular cartilage groups had small layers of CC thickness because of the severe erosions at the cartilage regions. The magnification of the image was $\times 400$. To accesses the degree of synovitis by HE staining, the $\mathrm{OA}$ and Articular cartilage groups showed the higher levels of cell layer, hyperplasia and cell infiltration in synovial membranes by compared with other groups. The magnification of image was $\times 200$. The synovial membrane of IHC stain was measured the expression level of IL-1 $\beta$. The high level of accumulation of cell layers and expression of IL-1 $\beta$ were observed in OA and Articular cartilage groups than other groups. The magnification of image was $\times 200$. All rats were $n=10$.

Table 2. Pathological articular cartilage and synovium analysis.

\begin{tabular}{|c|c|c|c|c|c|}
\hline Analysis & Sham & Meniscus & OA & $\mathbf{T}(\mathbf{M})$ & Articular cartilage \\
\hline \multicolumn{6}{|l|}{ Integrity of Cartilage } \\
\hline Cartilage damage & $0.40 \pm 0.24^{\mathrm{b}}$ & $0.20 \pm 0.00^{\mathrm{b}}$ & $5.40 \pm 0.40^{\mathrm{ac}}$ & $0.80 \pm 0.37 \mathrm{~b}$ & $5.20 \pm 0.20^{\mathrm{ac}}$ \\
\hline Loss of cellularity & $0.20 \pm 0.20^{\mathrm{bc}}$ & $0.40 \pm 0.25^{\mathrm{bc}}$ & $3.80 \pm 0.20^{\mathrm{ac}}$ & $1.40 \pm 0.24^{\mathrm{ab}}$ & $4.00 \pm 0.00^{\mathrm{ac}}$ \\
\hline Loss of matrix staining & $0.00 \pm 0.00^{\mathrm{bc}}$ & $0.00 \pm 0.00^{\mathrm{bc}}$ & $3.80 \pm 0.20 \mathrm{ac}$ & $2.00 \pm 0.45^{\mathrm{ab}}$ & $3.20 \pm 0.20 \mathrm{ac}$ \\
\hline Loss of tidemark integrity & $0.00 \pm 0.00^{\mathrm{b}}$ & $0.00 \pm 0.00^{\mathrm{b}}$ & $3.00 \pm 0.00 \mathrm{ac}$ & $0.40 \pm 0.24^{\mathrm{b}}$ & $2.60 \pm 0.24 \mathrm{ac}$ \\
\hline Modified Mankin Score & $0.60 \pm 0.25^{\mathrm{bc}}$ & $0.40 \pm 0.25^{\mathrm{bc}}$ & $28.00 \pm 1.05^{\mathrm{ac}}$ & $11.00 \pm 1.84^{\mathrm{ab}}$ & $27.00 \pm 0.55^{\mathrm{ac}}$ \\
\hline \multirow[t]{2}{*}{ UCC thickness $(\mu \mathrm{m})$} & $239.75 \pm 10.79 c$ & $232.89 \pm 9.66^{c}$ & meaningless & $130.39 \pm 39.03^{a}$ & meaningless \\
\hline & $297.18 \pm 23.14 \mathrm{c}$ & $293.90 \pm 24.06^{c}$ & meaningless & $135.06 \pm 24.44^{\mathrm{a}}$ & meaningless \\
\hline \multirow[t]{2}{*}{$\mathrm{CC}$ thickness $(\mu \mathrm{m})$} & $49.14 \pm 9.99 c$ & $45.68 \pm 15.32^{c}$ & meaningless & $163.83 \pm 37.88^{\mathrm{a}}$ & meaningless \\
\hline & $94.40 \pm 22.44^{c}$ & $101.06 \pm 15.96^{c}$ & meaningless & $165.96 \pm 40.20^{\mathrm{a}}$ & meaningless \\
\hline \multicolumn{6}{|l|}{ Grading of synovium } \\
\hline Lining of cell score & $0.40 \pm 0.25^{\mathrm{bc}}$ & $0.40 \pm 0.25^{\mathrm{bc}}$ & $2.60 \pm 0.25^{\mathrm{ac}}$ & $1.80 \pm 0.20 \mathrm{ab}$ & $2.80 \pm 0.20^{\mathrm{ac}}$ \\
\hline Presence of hyperplasia & $0.00 \pm 0.00^{\mathrm{bc}}$ & $0.00 \pm 0.00^{\mathrm{bc}}$ & $2.80 \pm 0.20 \mathrm{ac}$ & $1.60 \pm 0.25^{\mathrm{ab}}$ & $2.60 \pm 0.25^{\mathrm{ac}}$ \\
\hline Cell infiltration score & $0.00 \pm 0.00^{\mathrm{bc}}$ & $0.00 \pm 0.00^{\mathrm{bc}}$ & $2.80 \pm 0.20 \mathrm{ac}$ & $1.60 \pm 0.25^{\mathrm{ab}}$ & $2.60 \pm 0.25^{\mathrm{ac}}$ \\
\hline Synovitis score & $0.40 \pm 0.22^{\mathrm{bc}}$ & $0.40 \pm 0.22^{\mathrm{bc}}$ & $8.20 \pm 0.34^{\mathrm{ac}}$ & $5.00 \pm 0.40^{\mathrm{ab}}$ & $8.00 \pm 0.40^{\mathrm{ac}}$ \\
\hline IL-1 beta layer score & $0.33 \pm 0.17 \mathrm{bc}$ & $0.33 \pm 0.17 \mathrm{bc}$ & $2.22 \pm 0.22^{\mathrm{ac}}$ & $1.56 \pm 0.24^{\mathrm{ab}}$ & $2.33 \pm 0.20^{\mathrm{ac}}$ \\
\hline
\end{tabular}

For integrity of cartilage, mean \pm SEM obtained for all experimental groups with statistical results by Kruskal-Wallis test, except UCC and CC thicknesses were expressed as mean \pm SD with statistical results by one-way ANOVA. For grading of synovium, mean \pm SEM obtained for all experimental groups with statistical results by Kruskal-Wallis test, Statistical significance $\mathrm{p}<0.05 ; \mathrm{a}=$ Sham vs. other groups; $\mathrm{b}=\mathrm{OA}$ vs. other groups; $\mathrm{c}=\mathrm{T}(\mathrm{M})$ vs. other groups.

\section{Analysis of synovial membrane in joint after ESWT}

The histological changes of synovium in OA joint is characterized by the lining cells, increased mononuclear cell infiltration and blood vessel proliferation. In $\mathrm{T}(\mathrm{M})$ group, the histological evidences showed that the inflammatory synovitis was improved in the knee compared to OA and
Articular cartilage groups. There were significant differences in the lining of cell scores, synovial hyperplasia, cell infiltration scores and synovitis scores of Sham, Meniscus, and T(M) groups compared to $\mathrm{OA}$ and Articular cartilage groups $(P<0.05)$ (Figure 3: Synovitis and Table 2: Grading of synovium). Synovial membrane of IHC stains for IL-1 $\beta$ showed the hyperplasia of the cell layers and 


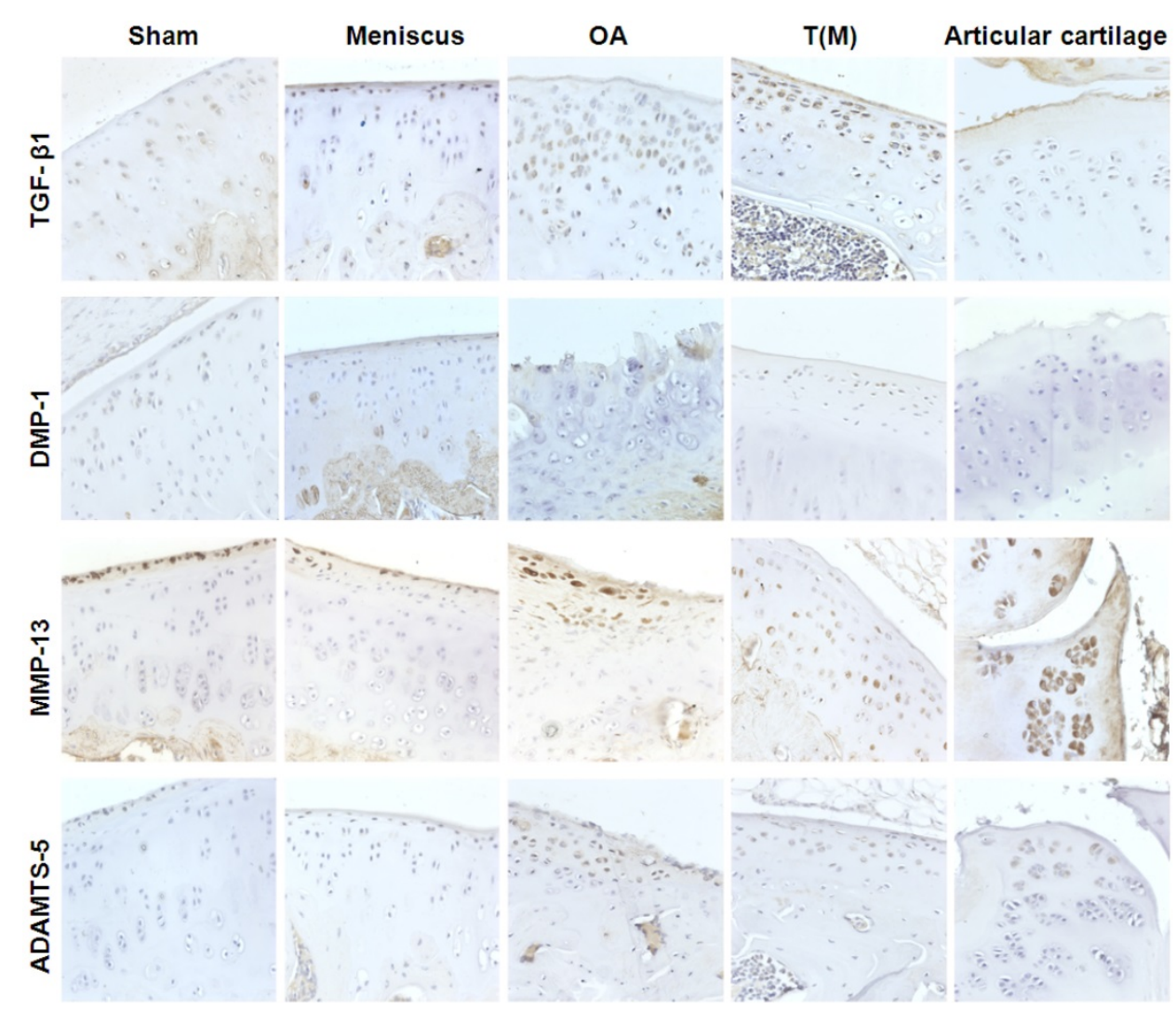

Figure 4. The therapeutic effects of ESWT on chondrogenesis proteins (TGF- $\beta 1$ and DMP-1) and cartilage degradation enzymes (MMP-13 and ADAMTS-5) in the cartilage regions of the knee joints. The IHC staining showed the expressions of TGF- $\beta 1$, DMP-1, MMP-13, and ADAMTS-5 in the each groups. The magnification of image was $\times 400$. All rats were $n=10$.

high level of IL-1 $\beta$ expression in OA and Articular cartilage groups but all were reduced in $\mathrm{T}(\mathrm{M})$ group. Further, IL-1 $\beta$ layer score was significant reduced in $\mathrm{T}(\mathrm{M})$ group by comparing with $\mathrm{OA}$ and Articular cartilage groups $(P<0.05)$ (Table 2$)$.

\section{Measurement of chondrogenesis and cartilage degradation factors}

The TGF- $\beta 1$ and DMP- 1 are directly related to the growth of the articular cartilage during development and cell proliferation. The expression level of TGF- $\beta 1$ and DMP-1 were measured by Immunohistochemical staining. Both factors were significantly increased in the T(M) group $(70 \pm 9.9 \%$ and $9 \pm 3.9 \%)$ compared to the OA $(46 \pm 9.4 \%$ and $4 \pm$ $1.9 \%)$ and Articular cartilage groups (52 $\pm 7.2 \%$ and 2 $\pm 2.5 \%)(\mathrm{P}<0.05)$ (Figure 4 as well as Figure $5 \mathrm{~A}$ and $5 B)$.

The matrix metalloproteinases (MMPs) and aggrecanases (ADAMTS) are the enzymes that degrade components of the cartilage extracellular matrix in OA knee. After ESWT treatment, MMP-13 and ADAMTS- 5 were measured and found significant decreased in $\mathrm{T}(\mathrm{M})$ group $(7 \pm 3.2 \%$ and $10 \pm 4.1 \%, P<$ $0.05)$ but no significant in Articular cartilage group (14 $\pm 2.4 \%$ and $21 \pm 5.2 \%$ ) by compared with OA group
(12 $\pm 2.8 \%$ and $24 \pm 7.9 \%$ ) (Figure 4 as well as Figure $5 \mathrm{C}$ and 5D).

\section{Discussion}

As opposed to the common treatment target of early osteoarthritis of the knee, the aims of the articular cartilage, the principal findings of the present study showed that the articular cartilage of early OA knee failed to respond to ESWT. Articular cartilage group showed no significant difference in pathological analysis, micro-CT analysis, cartilage grading score and grading of synovium by the comparison with OA group, except for $\mathrm{T}(\mathrm{M})$ group (subchondral bone) in the treatment of early OA knee (Table 1 and 2). In the immunohistochemical analysis, $\mathrm{T}(\mathrm{M})$ group significantly increased the expression of TGF- 31 and DMP-1 as well as reduced MMP-13 and ADAMTS- 5 in the cartilage of the tibia by compared with OA group and Articular cartilage group $(\mathrm{P}<0.05)$. Besides, in the comparison between Sham group and Meniscus group, we found that there were no any significant differences in OA lesion score, maximum extension angle, bone marrow density and medial tibia lesion (Table 1) that indicated the dosage of ESWT we use did not cause any damages in cartilage and subchondral bone. The results indicated 
A

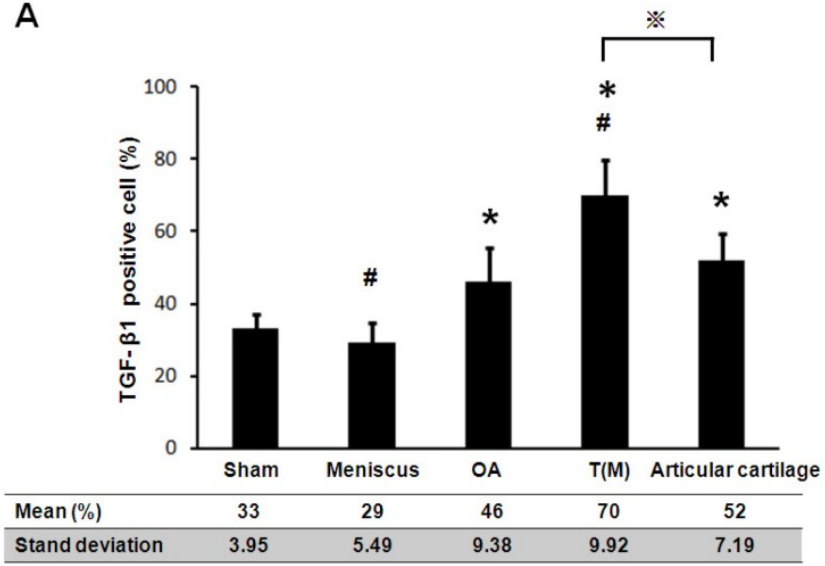

C

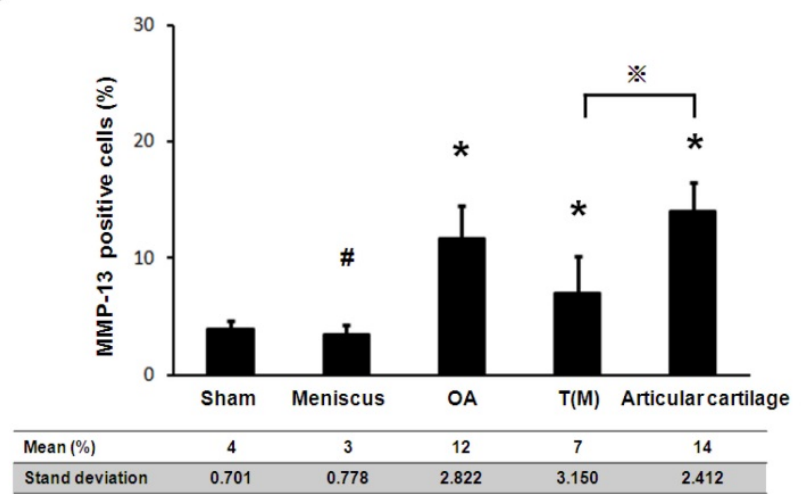

B

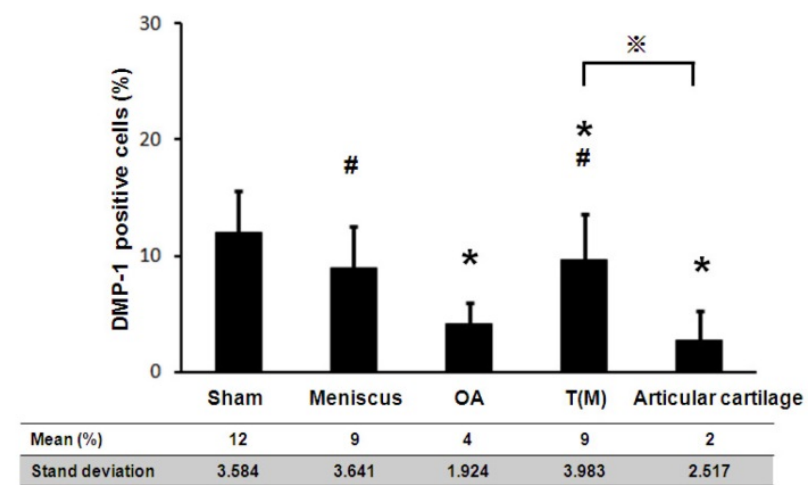

D

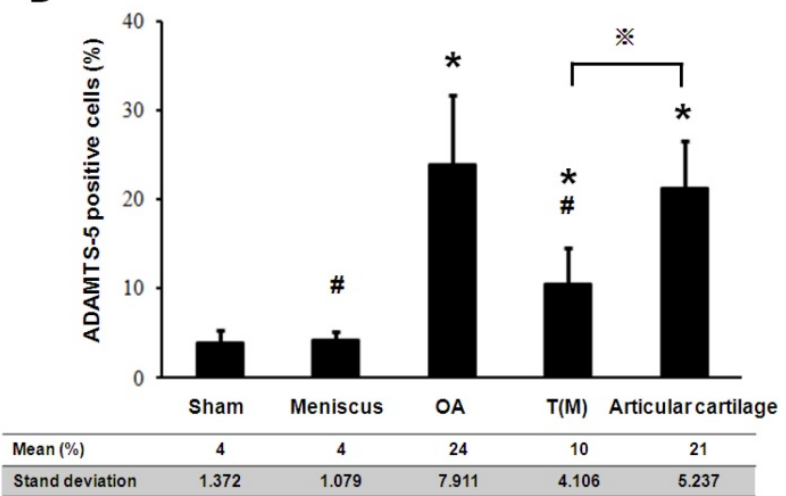

Figure 5. The expression level of TGF- $\beta 1$, DMP-1, MMP- 13 and ADAMTS- 5 were assessed in the percentage of positive cells. Presented the results mean \pm standard deviation (SD) of 10 rats in each group. The groups compared with Sham that was indicated the significant as $*(P<0.05)$ and compared with $O A$ to indicate as \# $(P<0.05)$ and compared with $T(M)$ to indicate as $※(p<0.05)$.

that the dosage of ESWT which we used was safe for the treatments. By the integration with previous study [29], we presumed that the optimal target area for ESWT is not the attenuated articular cartilage but the medial tibia subchondral bone in early osteoarthritis of the knee. With the effect of subchondral protection by ESWT, the progression of OA knee was retarded.

The present modalities for patients with early symptomatic osteoarthritis of the knee includes, participation in self-management programs [33,34], strengthening [35,36], low-impact aerobic exercises [37], neuromuscular education [38] and engage in physical activity under the supervision of physical therapist. Cortisone injection is proven for the reduction of pain via the strong anti-inflammatory effect, but repeated injection weakened the ligaments and tendon over time and might be jeopardized for the healthy cartilage [39]. Non-steroidal anti-inflammatory drugs (NSAIDs) can reduce pain, but long-term use can aggravate gastrointestinal irritation, blood pressure and risk of cardiovascular events [9]. Arthroscopic debridement, which is a more invasive procedure posed a mixed result, and may be no better than placebo [40]. Weight reduction and physical therapy were also reported to be beneficial. However, none of these aimed at the regeneration except popularly platelet-rich plasma (PRP) injection. According to a systematic review of preclinical studies and experiments, PRP application is proposed to prefer to use for knee treatment [41]. The researchers report that PRP treatment might cause part of changes in the joint environment and conduct short-term clinical improvements [42-44]. However, there still many biological factors should be concern about the clinical outcome and the optimize dosage of PRP injection for treatment of OA knee. Compared with PRP, ESWT poses the non-invasive nature and tissue regeneration potential with less operational variables.

With the emerging application of ESWT, it has been proposed to be beneficial for the early osteoarthritis of knee. The regression of osteoarthritis of rats knee were noticed after ESWT applied on the medial tibia [25]. Then, the medial tibia subchondral bone is identified as the key target for ESWT in early $\mathrm{OA}$ of the knee [22]. In present study, it is clarified that the articular cartilage is not an ideal target for ESWT, in contrast to other treatments which focus on the cartilaginous regeneration. The degenerative cartilage showed no significant difference in the 
comparison between OA group and Articular cartilage group but $\mathrm{T}(\mathrm{M})$ group showed significant regression (Figure 2). From the micro-CT analysis, the subchondral protection and regression is significantly noticed in T(M) group. Dr. Radin and his colleagues suggest the functional role of subchondral bone involving in the progression of early and late phase of OA knee [45]. Therefore, we postulated that the ESWT induced subchondral bone protection which leads to the subsequent articular cartilaginous protection instead of cartilaginous regeneration. Although the exact the mechanism of ESWT remained controversial, it is believed that the application of ESWT induced neovascularization and promotes angiogenesis and osteogenesis growth factors (eNOS, VEGF, PCNA, and BMP-2) that may lead to subchondral bone remodeling in terms of bone protection [20,46]. For the clinical application, ESWT on subchondral bone of knee instead of articular cartilage offers the protection or the retardation the progression of OA knee.

Limitations of the study exist. First, the outcome is based on the results of small animals. The physiology and anatomy of knee in rats may not necessarily resemble that of the human subject. Second, the dosage of ESWT remained unclear while applied on the human subject. Present study showed there is no cartilaginous damage induced by ESWT in this animal model but the safe and effective dosage for the human remained uncertain. Third, the timing of treatment intervention still needed to be defined. From the prevention of OA change of knee, the exact intervention age or knee conditions are unclear because a lot of factors, such as daily physical activity, body weight, muscular strength, ligamentous injury, fractures etc., are all affecting the development or the progression of OA knee. Therefore, with the present study and previous reports, future study should toward to the establishment of regimen of EWST in prevention, regression or retardation of osteoarthritic human knee.

\section{Conclusion}

This animal study showed that the articular cartilage is not a good target for ESWT on early knee osteoarthritis. We presumed that the optimal target area for ESWT is the medial tibial subchondral bone but not the attenuated articular cartilage in early osteoarthritis of the knee. With the effect of subchondral protection by ESWT, the progression of OA knee was retarded.

\section{Supplementary Material}

Supplementary figure.

http://www.medsci.org/v16p0156s1.pdf

\section{Acknowledgements}

We are grateful to the Department of Medical Research, Kaohsiung Chang Gung Memorial Hospital for the supporting of this work. Funds are received support for the research study presented in this article. The funding sources are from Chang Gung Medical Foundation (CMRPG8F1531, CMRPG8H0281 and CLRPG8E0131) and Ministry of Science and Technology (MOST 106-2314-B-182A-014).

\section{Competing interests}

The authors declared that they did not receive any honoraria or consultancy fees in writing this manuscript. No benefits in any form have been received or will be received from a commercial party related directly or indirectly to the subject of this article. One author (CJW) serves as a member of the advisory committee of SANUWAVE Health, Inc. (Suwanee, GA) and this study is performed independent of the appointment. The remaining authors declared no conflict of interest.

\section{References}

1. Willers C, Wood DJ, Zheng MH. A Current Review on the Biology and Treatment of Articular Cartilage Defects (Part I \& Part Ii). Journal of Musculoskeletal Research. 2003;07(03n04):157-181.

2. Lane NE, Nevitt MC. Osteoarthritis, bone mass, and fractures: how are they related? Arthritis Rheum 2002;46(1):1-4

3. Oettmeier R, Abendroth K. Osteoarthritis and bone: osteologic types of osteoarthritis of the hip. Skeletal Radiol. 1989;18(3):165-174.

4. Ratcliffe A, Seibel MJ. Biochemical markers of osteoarthritis. Curr Opin Rheumatol. 1990;2(5):770-776.

5. Hayami T, Funaki H, Yaoeda $\mathrm{K}$, et al. Expression of the cartilage derived anti-angiogenic factor chondromodulin-I decreases in the early stage of experimental osteoarthritis. J Rheumatol. 2003;30(10):2207-2217.

6. Stanitski CL. Articular Hypermobility and Chondral Injury in Patients With Acute Patellar Dislocation. The American Journal of Sports Medicine. 2016;23(2):146-150

7. Funck-Brentano T, Cohen-Solal M. Subchondral bone and osteoarthritis. Curr Opin Rheumatol. 2015;27(4):420-426.

8. Li G, Yin J, Gao J, et al. Subchondral bone in osteoarthritis: insight into risk factors and microstructural changes. Arthritis Research $\mathcal{E}$ Therapy. 2013;15(6):223.

9. Jevsevar DS. Treatment of Osteoarthritis of the Knee: Evidence-Based Guideline, 2nd Edition. Journal of the American Academy of Orthopaedic Surgeons. 2013;21(9):571-576.

10. Bhatia D, Bejarano T, Novo M. Current interventions in the management of knee osteoarthritis. Journal of Pharmacy and Bioallied Sciences. 2013;5(1):30.

11. Harris IA, Harris AM, Naylor JM, et al. Discordance Between Patient and Surgeon Satisfaction After Total Joint Arthroplasty. The Journal of Arthroplasty. 2013;28(5):722-727.

12. Schulze A, Scharf HP. Zufriedenheit nach Knietotalendoprothesenimplantation. Der Orthopäde. 2013;42(10):858-865.

13. Choi Y-J, Ra HJ. Patient Satisfaction after Total Knee Arthroplasty. Knee Surgery \& Related Research. 2016;28(1):1-15.

14. Falah M, Nierenberg G, Soudry M, et al. Treatment of articular cartilage lesions of the knee. International Orthopaedics. 2010;34(5):621-630.

15. Paschos NK. Recent advances and future directions in the management of knee osteoarthritis: Can biological joint reconstruction replace joint arthroplasty and when? World Journal of Orthopedics. 2015;6(9):655.

16. Jorge L. Escobar Ivirico MB, Emmanuel Kuyinu, Lakshmi S. Nair, Cato T. Laurencin. Regenerative Engineering for Knee Osteoarthritis Treatment: Biomaterials and Cell-Based Technologies. Engineering. 2017;3(1):16-27.

17. Ji Q Wang $\mathrm{P}, \mathrm{He} \mathrm{C}$. Extracorporeal shockwave therapy as a novel and potential treatment for degenerative cartilage and bone disease: Osteoarthritis. A qualitative analysis of the literature. Progress in Biophysics and Molecular Biology. 2016;121(3):255-265.

18. Wang CJ. An overview of shock wave therapy in musculoskeletal disorders. Chang Gung Med J. 2003;26(4):220-232.

19. Wang CJ. Extracorporeal shockwave therapy in musculoskeletal disorders. J Orthop Surg Res. 2012;7:11. 
20. Wang CJ, Wang FS, Yang KD, et al. Shock wave therapy induces neovascularization at the tendon-bone junction. A study in rabbits. J Orthop Res. 2003;21(6):984-989.

21. Wang CJ, Wang FS, Yang KD, et al. The effect of shock wave treatment at the tendon-bone interface-an histomorphological and biomechanical study in rabbits. J Orthop Res. 2005;23(2):274-280.

22. Wang CJ, Cheng JH, Huang CY, et al. Medial tibial subchondral bone is the key target for extracorporeal shockwave therapy in early osteoarthritis of the knee. Am J Transl Res. 2017;9(4):1720-1731.

23. Wang C-J, Cheng J-H, Chou W-Y, et al. Changes of articular cartilage and subchondral bone after extracorporeal shockwave therapy in osteoarthritis of the knee. International Journal of Medical Sciences. 2017;14(3):213-223.

24. Wang C-J, Sun Y-C, Siu K-K, et al. Extracorporeal shockwave therapy shows site-specific effects in osteoarthritis of the knee in rats. Journal of Surgical Research. 2013;183(2):612-619.

25. Wang C-J, Weng L-H, Ko J-Y, et al. Extracorporeal Shockwave Shows Regression of Osteoarthritis of the Knee in Rats. Journal of Surgical Research. 2011;171(2):601-608

26. Laverty S, Girard CA, Williams JM, et al. The OARSI histopathology initiative - recommendations for histological assessments of osteoarthritis in the rabbit. Osteoarthritis and Cartilage. 2010;18:S53-S65.

27. Hayami T, Pickarski M, Zhuo Y, et al. Characterization of articular cartilage and subchondral bone changes in the rat anterior cruciate ligament transection and meniscectomized models of osteoarthritis. Bone. 2006;38(2):234-243.

28. Wang C-J, Huang C-Y, Hsu S-L, et al. Extracorporeal shockwave therapy in osteoporotic osteoarthritis of the knee in rats: an experiment in animals. Arthritis Research \& Therapy. 2014;16(4):R139.

29. Salo PT, Hogervorst T, Seerattan RA, et al. Selective joint denervation promotes knee osteoarthritis in the aging rat. Journal of Orthopaedic Research. 2002;20(6):1256-1264.

30. Krenn V, Morawietz L, Burmester GR, et al. Synovitis score: discrimination between chronic low-grade and high-grade synovitis. Histopathology. 2006;49(4):358-364.

31. Cauli A, Yanni G, Panayi GS. Interleukin-1, interleukin-1 receptor antagonist and macrophage populations in rheumatoid arthritis synovial membrane. $\mathrm{Br} J$ Rheumatol. 1997;36(9):935-940.

32. Wang C-J, Weng L-H, Ko J-Y, et al. Extracorporeal shockwave therapy shows chondroprotective effects in osteoarthritic rat knee. Archives of Orthopaedic and Trauma Surgery. 2011;131(8):1153-1158.

33. Lorig K, Lubeck D, Kraines RG, et al. Outcomes of self-help education for patients with arthritis. Arthritis Rheum. 1985;28(6):680-685.

34. Coleman S, Briffa NK, Carroll G, et al. A randomised controlled trial of a self-management education program for osteoarthritis of the knee delivered by health care professionals. Arthritis Research \& Therapy. 2012;14(1):R21.

35. Fransen M, Crosbie J, Edmonds J. Physical therapy is effective for patients with osteoarthritis of the knee: a randomized controlled clinical trial. I Rheumatol. 2001;28(1):156-164.

36. Bennell KL, Hinman RS, Metcalf BR, et al. Efficacy of physiotherapy management of knee joint osteoarthritis: a randomised, double blind, placebo controlled trial. Ann Rheum Dis. 2005;64(6):906-912.

37. Kovar PA, Allegrante JP, MacKenzie CR, et al. Supervised fitness walking in patients with osteoarthritis of the knee. A randomized, controlled trial. Ann Intern Med. 1992;116(7):529-534.

38. Fitzgerald GK, Piva SR, Gil AB, et al. Agility and perturbation training techniques in exercise therapy for reducing pain and improving function in people with knee osteoarthritis: a randomized clinical trial. Phys Ther. 2011;91(4):452-469.

39. Dragoo JL, Danial CM, Braun HJ, et al. The chondrotoxicity of single-dose corticosteroids. Knee Surg Sports Traumatol Arthrosc. 2012;20(9):1809-1814.

40. Kirkley A, Birmingham TB, Litchfield RB, et al. A Randomized Trial of Arthroscopic Surgery for Osteoarthritis of the Knee. New England Journal of Medicine. 2008;359(11):1097-1107.

41. Filardo G, Kon E, Roffi A, et al. Platelet-rich plasma: why intra-articular? A systematic review of preclinical studies and clinical evidence on PRP for joint degeneration. Knee Surgery, Sports Traumatology, Arthroscopy. 2015;23(9):2459-2474.

42. van Buul GM, Koevoet WLM, Kops N, et al. Platelet-Rich Plasma Releasate Inhibits Inflammatory Processes in Osteoarthritic Chondrocytes. The American Journal of Sports Medicine. 2011;39(11):2362-2370.

43. Drengk A, Zapf A, Sturmer EK, et al. Influence of Platelet-Rich Plasma on Chondrogenic Differentiation and Proliferation of Chondrocytes and Mesenchymal Stem Cells. Cells Tissues Organs. 2009;189(5):317-326.

44. Anitua E, Sanchez M, Nurden AT, et al. Platelet-released growth factors enhance the secretion of hyaluronic acid and induce hepatocyte growth factor production by synovial fibroblasts from arthritic patients. Rheumatology. 2007:46(12):1769-1772

45. Radin EL, Rose RM. Role of subchondral bone in the initiation and progression of cartilage damage. Clin Orthop Relat Res. 1986;(213):34-40.

46. Cheng J-H, Wang C-J. Biological mechanism of shockwave in bone. International Journal of Surgery. 2015;24:143-146. 